curve of $S$ coincide, those of $\Sigma$ also are coincident. Moreover if the former constitute a plane curve, the tangent planes to $\Sigma$ along its flecnode curve, envelope a cone. But we have seen that this last is both necessary and sufficient to make the flecnode curve of $\Sigma$ a plane curve, for the envelope of the tangent planes along a branch of the flecnode curve is the secondary developable. We conclude therefore that if the two branches of the flecnode curve of a ruled surface coincide in a plane curve, the same is true of the dual surface. Moreover both surfaces have the dual property.

The University of Chicago, March, 1915.

\title{
ERRATA, VOLUME 15
}

Page 87. Burton H. Camp. Lebesgue integrals containing a parameter, with applications.

Page 106, line 2 from bottom, change last letter $\tau$ in line to $t_{1}$; last line, insert at end of sentence " and (a)." 